Research Article

\title{
Two Different Classes of Wronskian Conditions to a $(3+1)$-Dimensional Generalized Shallow Water Equation
}

\author{
Yaning Tang and Pengpeng Su \\ Department of Applied Mathematics, Northwestern Polytechnical University, \\ Shaanxi, Xi'an 710072, China \\ Correspondence should be addressed to Yaning Tang, tyaning@nwpu.edu.cn
}

Received 4 June 2012; Accepted 19 June 2012

Academic Editors: G. L. Karakostas and T. Ozawa

Copyright (C) 2012 Y. Tang and P. Su. This is an open access article distributed under the Creative Commons Attribution License, which permits unrestricted use, distribution, and reproduction in any medium, provided the original work is properly cited.

\begin{abstract}
Based on the Hirota bilinear method and Wronskian technique, two different classes of sufficient conditions consisting of linear partial differential equations system are presented, which guarantee that the Wronskian determinant is a solution to the corresponding Hirota bilinear equation of a $(3+1)$-dimensional generalized shallow water equation. Our results show that the nonlinear equation possesses rich and diverse exact solutions such as rational solutions, solitons, negatons, and positons.
\end{abstract}

\section{Introduction}

Wronskian formulations are a common feature for soliton equations, and it is a powerful tool to construct exact solutions to the soliton equations [1-4]. The resulting technique has been applied to many soliton equations such as the MKdV, NLS, derivative NLS, sine-Gordon, and other equations [5-10]. Within Wronskian formulations, soliton solutions, rational solutions, positons, and negatons are usually expressed as some kind of logarithmic derivatives of Wronskian-type determinants [11-16].

The following (3+1)-dimensional generalized shallow water equation

$$
u_{x x x y}-3 u_{x x} u_{y}-3 u_{x} u_{x y}+u_{y t}-u_{x z}=0
$$

was investigated in different ways (see, e.g., [17, 18]). In [17], soliton-typed solutions for (1.1) were constructed by a generalized tanh algorithm with symbolic computation. In [18], 
the traveling wave solutions of (1.1) expressed by hyperbolic, trigonometric, and rational functions were established by the $G^{\prime} / G$-expansion method, where $G=G(\xi)$ satisfies a second order linear ordinary differential equation.

Under a scale transformation $x \rightarrow-x,(1.1)$ is reduced equivalently to

$$
u_{x x x y}+3 u_{x x} u_{y}+3 u_{x} u_{x y}-u_{y t}-u_{x z}=0
$$

and the Cole-Hopf transformation

$$
u=2(\ln f)_{x}
$$

gives the corresponding Hirota bilinear equation of (1.2)

$$
\begin{aligned}
& \left(D_{x}^{3} D_{y}-D_{y} D_{t}-D_{x} D_{z}\right) f \cdot f \\
& \quad=\left(f_{x x x y}-f_{y t}-f_{x z}\right) f-f_{x x x} f_{y}-3 f_{x x y} f_{x}+f_{y} f_{t}+f_{x} f_{z}+3 f_{x x} f_{x y}=0,
\end{aligned}
$$

where $D_{x}, D_{y}, D_{z}$, and $D_{t}$ are the Hirota operators [19].

As we know, in the process of employing Wronskian technique, the main difficulty lies in looking for the linear differential conditions, which the functions in the Wronskian determinant should satisfy. Moreover, the differential conditions for the Wronskian determinant solutions of many soliton equations are not unique $[5,7,10,12]$. In this paper, we will give two different classes of linear differential conditions for the Nth order Wronskian determinant solutions (simply, Wronskian conditions) of (1.4) based on the special structure of the Hirota bilinear form (1.4). Our results will show that (1.4) has diverse Wronskian determinant solutions under different linear differential conditions and further (1.2) will have diverse exact solutions such as rational solutions, solitons, negatons, and positons.

\section{The First Class of Wronskian Conditions}

The Nth order Wronskian determinant was introduced firstly by Freeman and Nimmo [1, 20]:

$$
W\left(\phi_{1}, \phi_{2}, \ldots, \phi_{N}\right)=|\widehat{N-1}|=\left|\begin{array}{cccc}
\phi_{1}^{(0)} & \phi_{1}^{(1)} & \ldots & \phi_{1}^{(N-1)} \\
\phi_{2}^{(0)} & \phi_{2}^{(1)} & \ldots & \phi_{2}^{(N-1)} \\
\vdots & \vdots & \ddots & \vdots \\
\phi_{N}^{(0)} & \phi_{N}^{(1)} & \ldots & \phi_{N}^{(N-1)}
\end{array}\right|, \quad N \geq 1
$$

where

$$
\phi_{i}^{(j)}=\frac{\partial^{j}}{\partial x^{j}} \phi_{i}, \quad 0 \leq j \leq N-1,1 \leq i \leq N
$$

Solutions determined by $f=|\widehat{N-1}|$ to (1.4) are called Wronskian determinant solutions. 
In this section, we present the first class of linear differential conditions for the Wronskian determinant solutions of (1.4).

Theorem 2.1. Let a set of functions $\phi_{i}=\phi_{i}(x, y, z, t), 1 \leq i \leq N$ satisfy the following linear differential conditions:

$$
\begin{gathered}
\phi_{i, x x}=\sum_{j=1}^{N} \lambda_{i j} \phi_{j}, \\
\phi_{i, y}=n \phi_{i, x}, \\
\phi_{i, t}=-\phi_{i,(m x),} \\
\phi_{i, z}=4 n \phi_{i, x x x}+n \phi_{i,(m x),}
\end{gathered}
$$

where the coefficient matrix $A=\left(\lambda_{i j}\right)_{1 \leq i, j \leq N}$ is an arbitrary real constant matrix (see [10, 12]), $n$ is an arbitrary nonzero constant, $m$ is an arbitrary positive integer, and $\phi_{i,(m x)}$ denotes the $m$ th order derivative of $\phi_{i}$ with respect to $x$. Then the Wronskian determinant $f=|\widehat{N-1}|$ defined by (2.1) solves (1.4).

The proof of Theorem 2.1 needs the following two useful known Lemmas.

Lemma 2.2. Set $a_{j}, j=1,2, \ldots, N$ to be an $N$-dimensional column vector, and $b_{j}, j=1,2, \ldots, N$ to be a real nonzero constant. Then one has

$$
\sum_{i=1}^{N} b_{i}\left|a_{1}, a_{2}, \ldots, a_{N}\right|=\sum_{j=1}^{N}\left|a_{1}, a_{2}, \ldots, b a_{j}, \ldots, a_{N}\right|
$$

where $b a_{j}=\left(b_{1} a_{1 j}, b_{2} a_{2 j}, \ldots, b_{N} a_{N j}\right)^{T}$.

Lemma 2.3 (see [11]). Under the condition (2.3) and Lemma 2.2, the following equalities hold:

$$
\begin{aligned}
|\widehat{N-1}| \sum_{i=1}^{N} \lambda_{i i}\left(\sum_{i=1}^{N} \lambda_{i i}|\widehat{N-1}|\right) \\
=\left(\sum_{i=1}^{N} \lambda_{i i}|\widehat{N-1}|\right)^{2} \\
=(-|\widehat{N-3}, N-1, N|+|\widehat{N-2}, N+1|)^{2} \\
=|\widehat{N-1}|(|\widehat{N-5}, N-3, N-2, N-1, N|-|\widehat{N-4}, N-2, N-1, N+1| \\
\quad+2|\widehat{N-3}, N, N+1|-|\widehat{N-3}, N-1, N+2|+|\widehat{N-2}, N+3|) .
\end{aligned}
$$


Proof of Theorem 2.1. Under the properties of the Wronskian determinant and the conditions (2.3) and (2.4), we can compute various derivatives of the Wronskian determinant $f=|\widehat{N-1}|$ with respect to the variables $x, y, z, t$ as follows:

$$
\begin{gathered}
f_{x}=|\widehat{N-2}, N|, \quad f_{x x}=|\widehat{N-3}, N-1, N|+|\widehat{N-2}, N+1|, \\
f_{x x x}=|\widehat{N-4}, N-2, N-1, N|+2|\widehat{N-3}, N-1, N+1|+|\widehat{N-2}, N+2|, \\
f_{y}=n|\widehat{N-2}, N|, \quad f_{x y}=n(|\widehat{N-3}, N-1, N|+|\widehat{N-2}, N+1|), \\
f_{x x y}=n(|\widehat{N-4}, N-2, N-1, N|+2|\widehat{N-3}, N-1, N+1|+|\widehat{N-2}, N+2|), \\
f_{x x x y}=n(|\widehat{N-5}, N-3, N-2, N-1, N|+3|\widehat{N-4}, N-2, N-1, N+1| \\
\quad+2|\widehat{N-3}, N, N+1|+3|\widehat{N-3}, N-1, N+2|+|\widehat{N-2}, N+3|) \\
f_{t}=-\frac{\partial^{m} f}{\partial x^{m}}=-\frac{\partial^{m}|\widehat{N-1}|}{\partial x^{m}}, \quad f_{y t}=-\frac{\partial}{\partial y} \frac{\partial^{m}|\widehat{N-1}|}{\partial x^{m}}=-n \frac{\partial}{\partial x} \frac{\partial^{m}|\widehat{N-1}|}{\partial x^{m}}, \\
f_{z=} 4 n(|\widehat{N-4}, N-2, N-1, N|-|\widehat{N-3}, N-1, N+1|+|\widehat{N-2}, N+2|) \\
+n \frac{\partial^{m}|\widehat{N-1}|}{\partial x^{m}}, \\
+n \frac{\partial}{\partial x} \frac{\partial^{m}|\widehat{N-1}|}{\partial x^{m}} . \\
f_{x z=}
\end{gathered}
$$

Therefore, we can now compute that

$$
\begin{aligned}
\left(f_{x x x y}-f_{y t}-f_{x z}\right) f & \\
= & 3 n(-|\widehat{N-5}, N-3, N-2, N-1, N|+|\widehat{N-4}, N-2, N-1, N+1| \\
& \quad+2|\widehat{N-3}, N, N+1|+|\widehat{N-3}, N-1, N+2|-|\widehat{N-2}, N+3|)|\widehat{N-1}|, \\
& \quad-f_{x x x} f_{y}-3 f_{x x y} f_{x}+f_{y} f_{t}+f_{x} f_{z} \\
= & -12 n|\widehat{N-3}, N-1, N+1||\widehat{N-2}, N|,
\end{aligned}
$$




$$
\begin{aligned}
3 f_{x x} & f_{x y} \\
& =3 n(|\widehat{N-3}, N-1, N|+|\widehat{N-2}, N+1|)^{2} \\
& =3 n(-|\widehat{N-3}, N-1, N|+|\widehat{N-2}, N+1|)^{2}+12 n|\widehat{N-3}, N-1, N||\widehat{N-2}, N+1| .
\end{aligned}
$$

Using Lemma 2.3, we can further obtain that

$$
\begin{aligned}
\left(D_{x}^{3} D_{y}-D_{y} D_{t}-D_{x} D_{z}\right) f \cdot f \\
=12 n(|\widehat{N-3}, N, N+1||\widehat{N-1}|-|\widehat{N-3}, N-1, N+1||\widehat{N-2}, N| \\
\quad+|\widehat{N-3}, N-1, N||\widehat{N-2}, N+1|) \\
=0 .
\end{aligned}
$$

This last equality is just the Plücker relation for determinants:

$$
\left|B, A_{1}, A_{2}\right|\left|B, A_{3}, A_{4}\right|-\left|B, A_{1}, A_{3}\right|\left|B, A_{2}, A_{4}\right|+\left|B, A_{1}, A_{4}\right|\left|B, A_{2}, A_{3}\right|=0,
$$

where $B$ denotes an $N \times(N-2)$ matrix, and $A_{i}, 1 \leq i \leq 4$ are four $N$-dimensional column vectors. Therefore, we have shown that $f=|\widehat{N-1}|$ solve (1.4) under the conditions (2.3) and (2.4). Further, the corresponding solution to (1.2) is

$$
u=2(\ln f)_{x^{\prime}}, \quad f=|\widehat{N-1}| .
$$

Remarks 1. The condition (2.4) is a generalized linear differential condition which includes many different special cases.

For example, when $m=1$, the condition (2.4) is reduced to

$$
\phi_{i, y}=n \phi_{i, x}, \quad \phi_{i, t}=-\phi_{i, x}, \quad \phi_{i, z}=4 n \phi_{i, x x x}+n \phi_{i, x} .
$$

When $m=2$, the condition (2.4) is reduced to

$$
\phi_{i, y}=n \phi_{i, x}, \quad \phi_{i, t}=-\phi_{i, x x}, \quad \phi_{i, z}=4 n \phi_{i, x x x}+n \phi_{i, x x} .
$$

When $m=3$, the condition (2.4) is reduced to

$$
\phi_{i, y}=n \phi_{i, x}, \quad \phi_{i, t}=-\phi_{i, x x x}, \quad \phi_{i, z}=5 n \phi_{i, x x x} .
$$


Using the linear differential conditions (2.3) and (2.4) as well as the transformation (1.3), we can compute many exact solutions of (1.2) such as rational solutions, solitons, negatons, and positons.

As an example, in the special case of $m=2$ and $n=1$, the conditions (2.3) and (2.4) read

$$
\begin{gathered}
\phi_{i, x x}=\sum_{j=1}^{N} \lambda_{i j} \phi_{j}, \quad i=1, \ldots, N, \\
\phi_{i, y}=\phi_{i, x}, \\
\phi_{i, t}=-\phi_{i, x x} \\
\phi_{i, z}=4 \phi_{i, x x x}+\phi_{i, x x} .
\end{gathered}
$$

If we let the coefficient matrix $A=\left(\lambda_{i j}\right)_{1 \leq i, j \leq N}$ of condition (2.15) has the following form (see $[10-12,16]$ for details),

$$
A=\left(\begin{array}{cccc}
\lambda_{11} & & & 0 \\
& \lambda_{22} & & \\
& & \ddots & \\
0 & & & \lambda_{N N}
\end{array}\right)_{N \times N}, \quad\left(\lambda_{i i} \neq \lambda_{j j}, i \neq j\right)
$$

using the same method as that in [16], we can obtain $N$-soliton solutions of (1.2).

For example, when $\lambda_{11}>0, \lambda_{i i}=0, i=2, \ldots, N$, we can compute two exact 1 -soliton solutions for (1.2),

$$
\begin{aligned}
u_{1} & =2 \partial_{x} \ln \left(\cosh \left(4 \lambda_{11}^{3 / 2} z+\lambda_{11} z+\sqrt{\lambda_{11}} y+\sqrt{\lambda_{11}} x-\lambda_{11} t+\gamma_{1}\right)\right) \\
& =2 \sqrt{\lambda_{11}} \tanh \left(4 \lambda_{11}^{3 / 2} z+\lambda_{11} z+\sqrt{\lambda_{11}} y+\sqrt{\lambda_{11}} x-\lambda_{11} t+\gamma_{1}\right), \\
u_{2} & =2 \partial_{x} \ln \left(\sinh \left(4 \lambda_{11}^{3 / 2} z+\lambda_{11} z+\sqrt{\lambda_{11}} y+\sqrt{\lambda_{11}} x-\lambda_{11} t+\gamma_{1}\right)\right) \\
& =2 \sqrt{\lambda_{11}} \operatorname{coth}\left(4 \lambda_{11}^{3 / 2} z+\lambda_{11} z+\sqrt{\lambda_{11}} y+\sqrt{\lambda_{11}} x-\lambda_{11} t+\gamma_{1}\right),
\end{aligned}
$$

with $\gamma_{1}$ being a constant.

\section{The Second Class of Wronskian Condition}

In this section, we show another linear differential condition to the Wronskian determinant solutions of (1.4). 
Theorem 3.1. Let a group of functions $\phi_{i}=\phi_{i}(x, y, z, t), 1 \leq i \leq N$ satisfy the following linear differential condition:

$$
\begin{gathered}
\phi_{i, y}=k \phi_{i, x x}, \\
\phi_{i, t}=-2 \phi_{i, x x x}, \\
\phi_{i, z}=3 k \phi_{i, x x x x}
\end{gathered}
$$

where $k$ is an arbitrary nonzero constant. Then the Wronskian determinant $f=|\widehat{N-1}|$ defined by (2.1) solves (1.4).

Proof. Under the properties of the Wronskian determinant and the condition (3.1), various derivatives of the Wronskian determinant $f=|\widehat{N-1}|$ with respect to the variables $x, y, z, t$ are obtained as follows:

$$
\begin{aligned}
& f_{x}=|\widehat{N-2}, N|, \quad f_{x x}=|\widehat{N-3}, N-1, N|+|\widehat{N-2}, N+1|, \\
& f_{x x x}=|\widehat{N-4}, N-2, N-1, N|+2|\widehat{N-3}, N-1, N+1|+|\widehat{N-2}, N+2|, \\
& f_{y}=k(-|\widehat{N-3}, N-1, N|+|\widehat{N-2}, N+1|), \\
& f_{x y}=k(-|\widehat{N-4}, N-2, N-1, N|+|\widehat{N-2}, N+2|), \\
& f_{x x y}=k(-|\widehat{N-5}, N-3, N-2, N-1, N|-|\widehat{N-4}, N-2, N-1, N+1| \\
& +|\widehat{N-3}, N-1, N+2|+|\widehat{N-2}, N+3|), \\
& f_{x x x y}=k(-|\widehat{N-6}, N-4, N-3, N-2, N-1, N|-|\widehat{N-4}, N-2, N, N+1| \\
& -2|\widehat{N-5}, N-3, N-2, N-1, N+1|+|\widehat{N-3}, N, N+2| \\
& +2|\widehat{N-3}, N-1, N+3|+|\widehat{N-2}, N+4|), \\
& f_{t}=-2(|\widehat{N-4}, N-2, N-1, N|-|\widehat{N-3}, N-1, N+1|+|\widehat{N-2}, N+2|) \text {, } \\
& f_{y t}=-2 k(-|\widehat{N-6}, N-4, N-3, N-2, N-1, N|-|\widehat{N-4}, N-2, N, N+1| \\
& +|\widehat{N-5}, N-3, N-2, N-1, N+1|+|\widehat{N-3}, N, N+2| \\
& -|\widehat{N-3}, N-1, N+3|+|\widehat{N-2}, N+4|), \\
& f_{z}=3 k(-|\widehat{N-5}, N-3, N-2, N-1, N|+|\widehat{N-4}, N-2, N-1, N+1| \\
& -|\widehat{N-3}, N-1, N+2|+|\widehat{N-2}, N+3|),
\end{aligned}
$$




$$
\begin{gathered}
f_{x z}=3 k(-|\widehat{N-6}, N-4, N-3, N-2, N-1, N|+|\widehat{N-4}, N-2, N, N+1| \\
-|\widehat{N-3}, N, N+2|+|\widehat{N-2}, N+4|) .
\end{gathered}
$$

Therefore, we can now compute that

$$
\begin{gathered}
\left(f_{x x x y}-f_{y t}-f_{x z}\right) f=6 k(-|\widehat{N-4}, N-2, N, N+1|+|\widehat{N-3}, N, N+2|)|\widehat{N-1}|, \\
-3 f_{x x y} f_{x}+f_{x} f_{z}=6 k(|\widehat{N-4}, N-2, N-1, N+1|-|\widehat{N-3}, N-1, N+2|)|\widehat{N-2}, N|, \\
-f_{x x x} f_{y}+f_{y} f_{t}+3 f_{x x} f_{x y}=6 k(-|\widehat{N-4}, N-2, N-1, N||\widehat{N-2}, N+1| \\
+|\widehat{N-3}, N-1, N||\widehat{N-2}, N+2|),
\end{gathered}
$$

then, substituting the above results into (1.4), we can further obtain that

$$
\left(D_{x}^{3} D_{y}-D_{y} D_{t}-D_{x} D_{z}\right) f \cdot f=6 k\left(A_{1}+A_{2}\right),
$$

where

$$
\begin{aligned}
A_{1}= & -|\widehat{N-4}, N-2, N, N+1||\widehat{N-1}|+|\widehat{N-4}, N-2, N-1, N+1||\widehat{N-2}, N| \\
& -|\widehat{N-4}, N-2, N-1, N||\widehat{N-2}, N+1| \\
= & |\widehat{N-4}, N-2, N-3, N-1||\widehat{N-4}, N-2, N, N+1| \\
& -|\widehat{N-4}, N-2, N-3, N||\widehat{N-4}, N-2, N-1, N+1| \\
& +|\widehat{N-4}, N-2, N-3, N+1||\widehat{N-4}, N-2, N-1, N|, \\
A_{2}= & |\widehat{N-3}, N, N+2||\widehat{N-1}|-|\widehat{N-3}, N-1, N+2||\widehat{N-2}, N| \\
& +|\widehat{N-3}, N-1, N||\widehat{N-2}, N+2| \\
= & |\widehat{N-3}, N, N+2||\widehat{N-3}, N-2, N-1| \\
& -|\widehat{N-3}, N-2, N||\widehat{N-3}, N-1, N+2| \\
& +|\widehat{N-3}, N-2, N+2||\widehat{N-3}, N-1, N| .
\end{aligned}
$$


From (3.5), it is easy to see that the above expression (3.4) is nothing but zero because they both satisfy the Plücker relation (2.10). Therefore, we have shown that $f=|\widehat{N-1}|$ also solve (1.4) under the condition (3.1).

The condition (3.1) has an exponential-type function solution:

$$
\phi_{i}=\sum_{j=1}^{p} d_{i j} e^{\eta_{i j}}, \quad \eta_{i j}=l_{i j} x+k l_{i j}^{2} y+3 k l_{i j}^{4} z-2 l_{i j}^{3} t, \quad i=1,2, \ldots, N,
$$

where $d_{i j}$ and $l_{i j}$ are free parameters and $p$ is an arbitrary natural number.

In particular, we can have the following Wronskian solutions of (1.2):

$$
u=2(\ln f)_{x^{\prime}} \quad f=W\left(\phi_{1}, \phi_{2}, \ldots, \phi_{N}\right),
$$

where

$$
\phi_{i}=e^{l_{i} x+k l_{i}^{2} y+3 k l_{i}^{4} z-2 l_{i}^{3} t}+e^{w_{i} x+k w_{i}^{2} y+3 k w_{i}^{4} z-2 w_{i}^{3} t}, \quad i=1, \ldots, N,
$$

with $l_{i}$ and $w_{i}$ being free parameters.

\section{Conclusions and Remarks}

In summary, we have established two different kinds of linear differential conditions for the Wronskian determinant solutions of the $(3+1)$-dimensional generalized shallow water equation (1.1) or equivalently (1.2). Especially, the first Wronskian conditions are generalized linear differential conditions which include many different special cases. Our results show that the nonlinear equation (1.1) carry rich and diverse Wronskian determinant solutions.

\section{Acknowledgments}

This work was supported in part by the National Science Foundation of China (under Grant nos. 11172233, 11102156, and 11002110) and Northwestern Polytechnical University Foundation for Fundamental Research (no. GBKY1034).

\section{References}

[1] N. C. Freeman and J. J. C. Nimmo, "Soliton solutions of the Korteweg-de Vries and KadomtsevPetviashvili equations: the Wronskian technique," Physics Letters A, vol. 95, no. 1, pp. 1-3, 1983.

[2] V. B. Matveev, "Positon-positon and soliton-positon collisions: KdV case," Physics Letters A, vol. 166, no. 3-4, pp. 209-212, 1992.

[3] W.-X. Ma, "Wronskians, generalized Wronskians and solutions to the Korteweg-de Vries equation," Chaos, Solitons \& Fractals, vol. 19, no. 1, pp. 163-170, 2004.

[4] W.-X. Ma and K.-i. Maruno, "Complexiton solutions of the Toda lattice equation," Physica A, vol. 343, no. 1-4, pp. 219-237, 2004.

[5] X. Geng and Y. Ma, "N-soliton solution and its Wronskian form of a $(3+1)$-dimensional nonlinear evolution equation," Physics Letters A, vol. 369, no. 4, pp. 285-289, 2007. 
[6] Z. Yan, "Multiple solution profiles to the higher-dimensional Kadomtsev-Petviashvilli equations via Wronskian determinant," Chaos, Solitons and Fractals, vol. 33, no. 3, pp. 951-957, 2007.

[7] J. Wu, "N-soliton solution, generalized double Wronskian determinant solution and rational solution for a $(2+1)$-dimensional nonlinear evolution equation," Physics Letters A, vol. 373, no. 1, pp. 83-88, 2008.

[8] J. Ji, "The double Wronskian solutions of a non-isospectral Kadomtsev-Petviashvili equation," Physics Letters A, vol. 372, no. 39, pp. 6074-6081, 2008.

[9] Y. Zhang, Y.-N. Lv, L.-Y. Ye, and H.-Q. Zhao, "The exact solutions to the complex KdV equation," Physics Letters A, vol. 367, no. 6, pp. 465-472, 2007.

[10] C.-X. Li, W.-X. Ma, X.-J. Liu, and Y.-B. Zeng, "Wronskian solutions of the Boussinesq equationsolitons, negatons, positons and complexitons," Inverse Problems, vol. 23, no. 1, pp. 279-296, 2007.

[11] W.-X. Ma and Y. You, "Solving the Korteweg-de Vries equation by its bilinear form: Wronskian solutions," Transactions of the American Mathematical Society, vol. 357, no. 5, pp. 1753-1778, 2005.

[12] W.-X. Ma, C.-X. Li, and J. He, "A second Wronskian formulation of the Boussinesq equation," Nonlinear Analysis A, vol. 70, no. 12, pp. 4245-4258, 2009.

[13] W.-X. Ma, "Wronskian solutions to integrable equations," Discrete and Continuous Dynamical Systems A, pp. 506-515, 2009.

[14] X. Geng, "Algebraic-geometrical solutions of some multidimensional nonlinear evolution equations," Journal of Physics A, vol. 36, no. 9, pp. 2289-2303, 2003.

[15] J. P. Wu, "A new Wronskian condition for a (3+1)-dimensional nonlinear evolution equation," Chinese Physics Letters, vol. 28, no. 5, article 050501, 2011.

[16] Y. Tang, W.-X. Ma, W. Xu, and L. Gao, "Wronskian determinant solutions of the (3+1)-dimensional Jimbo-Miwa equation," Applied Mathematics and Computation, vol. 217, no. 21, pp. 8722-8730, 2011.

[17] B. Tian and Y. T. Gao, "Beyond traveling waves: a new algorithm for solving nonlinear evolution equations," Computer Physics Communications, vol. 95, pp. 39-142, 1996.

[18] E.M.E. Zayed, "Traveling wave solutions for higher dimensional nonlinear evolution equations using the $G^{\prime}$ / G-expansion method," Journal of Applied Mathematics \& Informatics, vol. 28, pp. 383-395, 2010.

[19] R. Hirota, The Direct Methods in Soliton Theory, vol. 155 of Cambridge Tracts in Mathematics, Cambridge University Press, Cambridge, UK, 2004.

[20] J. J. C. Nimmo and N. C. Freeman, "A method of obtaining the $N$-soliton solution of the Boussinesq equation in terms of a Wronskian," Physics Letters A, vol. 95, no. 1, pp. 4-6, 1983. 


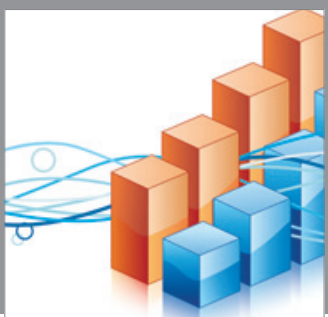

Advances in

Operations Research

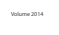

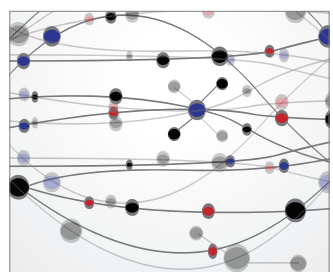

\section{The Scientific} World Journal
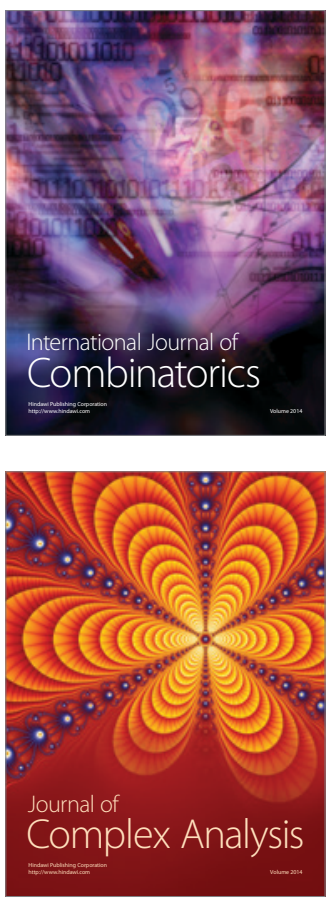

International Journal of

Mathematics and

Mathematical

Sciences
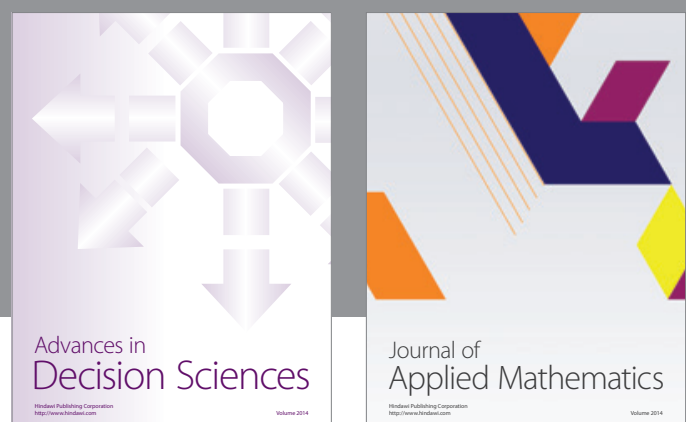

Journal of

Applied Mathematics
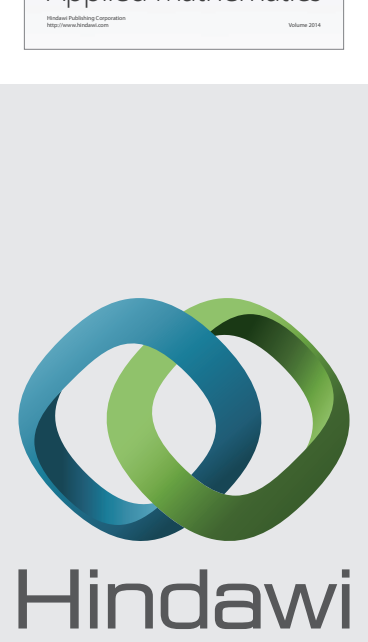

Submit your manuscripts at http://www.hindawi.com
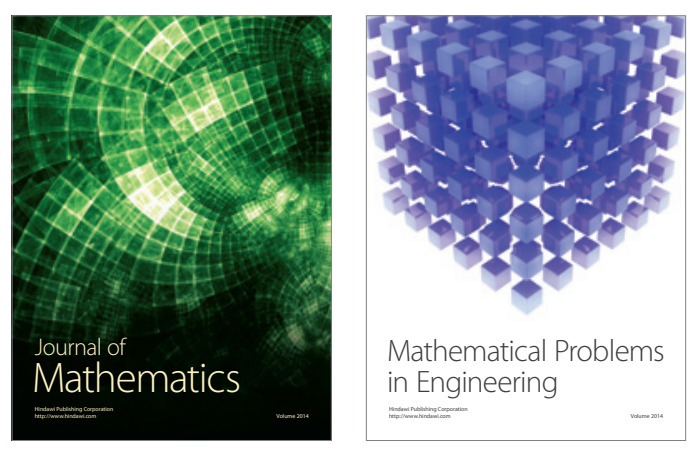

Mathematical Problems in Engineering
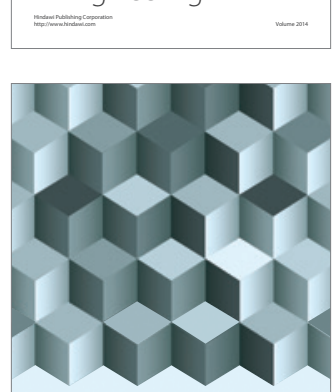

Journal of

Function Spaces
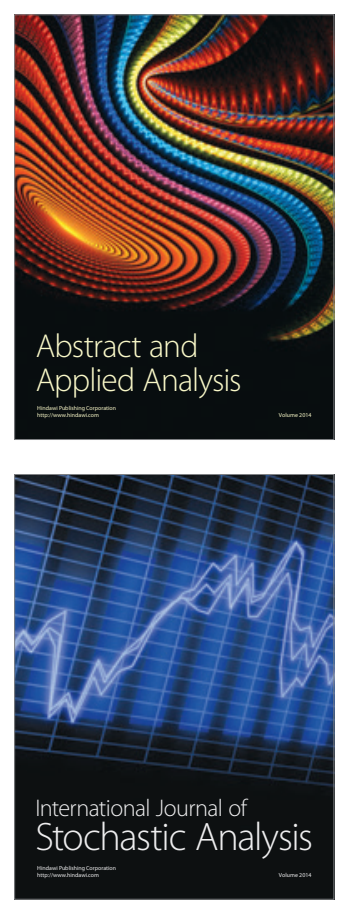

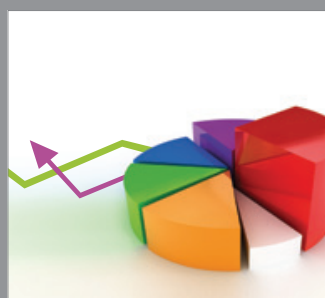

ournal of

Probability and Statistics

Promensencen
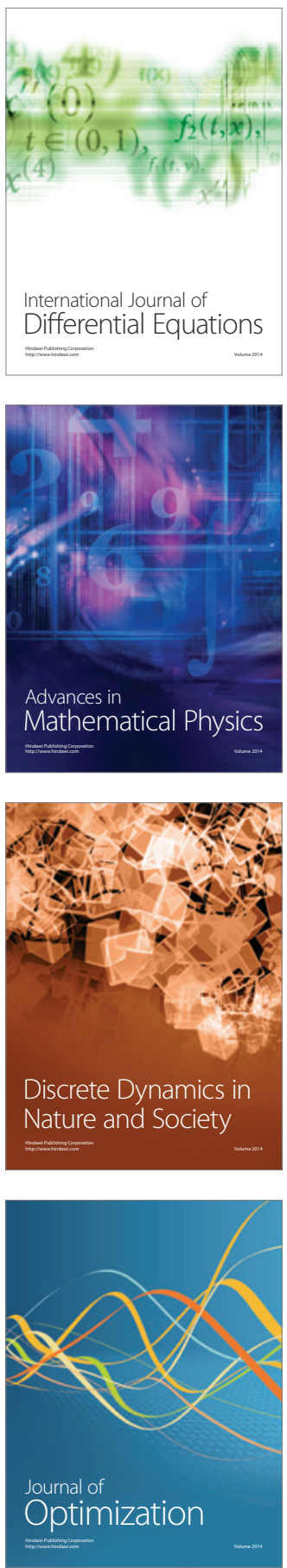